\title{
Wet Granulation to Overcome Liquisolid Technique Issues of Poor Flowability and Compactibility: A Study to Enhance Glibenclamide Dissolution
}

\author{
Javaheri H, Carter P and Elkordy AA* \\ Department of Pharmacy, Health and Well-being, University of Sunderland, Sunderland, SR1 3SD, UK
}

${ }^{*}$ Corresponding author: Elkordy AA, Department of Pharmacy, Health and Well-being, University of Sunderland, Sunderland, SR1 3SD, UK, Fax: 0044 (0) 1915153405, Tel: 0044 (0) 1915152576, E-mail: amal. elkordy@sunderland.ac.uk

Citation: Javaheri H, Carter P, Elkordy AA (2014) Wet Granulation to Overcome Liquisolid Technique Issues of Poor Flowability and Compactibility: A Study to Enhance Glibenclamide Dissolution. J Pharm Drug Devel 2(3): 301. doi: 10.15744/2348-9782.1.501

\section{Received Date: August 25, 2014 Accepted Date: October 19, 2014 Published Date: October 21, 2014}

\begin{abstract}
The aim of this study is to apply wet granulation on liquisolid powders to overcome issues of poor powder flowability and compressibility especially with using high viscosity liquid vehicles. Different liquisolid formulations were made using three excipients where the effect of each excipient used in the dissolution of the model hydrophobic drug (Glibenclamide) was evaluated. The Glibenclamide tablets were formulated using PEG 400, Synperonic PE/L44 and Cremophor ELP, at a $10 \% \mathrm{w} / \mathrm{w}$ in liquid vehicle drug concentration. The carrier (Avicel ${ }^{\circ} \mathrm{PH} 102$ ) was used followed by colloidal silicon dioxide (coating material) that converted the wet mixture into dry powder. Potato starch, $5 \% \mathrm{w} / \mathrm{w}$, as a disintegrant was mixed with the mixture manually for 10 minutes and was finalized by adding $0.75 \%$ of magnesium stearate as a lubricant. The final powder (depending on its flowability and compactability) was then compacted automatically using a single-punch tableting machine to give tablets with $4 \mathrm{mg}$ unit drug dose. Prepared liquisolid compacts were characterised via B.P. quality control tests. In this study, a novel discovery was achieved to overcome the major problems with liquisolid preparations (flowability and compactability). This new technique is the wet granulation process to be applied with liquisolid powders just before the compaction stage of the powders into tablets. Consequently, it was found that by application of wet granulation to liquisolid powder admixture, the large-scale production of liquisolid compacts is feasible and can be easily applied by pharmaceutical industry. As with some liquisolid vehicles especially high viscosity although very good to enhance dissolution usually formulations are studied in powder forms [1,2].
\end{abstract}

In addition, the In-vitro dissolution study found that granulated liquisolid tablets enhanced not only the flowability and compressibility of the powder drug, but also enhanced drug dissolution due to the increased wetting properties of hydrophobic drug making it more available for dissolution.

Keywords: Liquisolid compacts; Granulation; Glibenclamide; Flowability and compactibility; Dissolution rate; Wettability

\section{Introduction}

Oral drug delivery is the main and most common route of administration. There are various types of dosage forms that can be formulated, and tablets are known to be the most common type. Tablets can be manufactured by several stages where the final stage is the compression of the powder held within a confined space. In addition, the use of tablets as dosage form became of interest to the pharmaceutical industry, thus the formulation of tablet with variety of forms were formulated [3]. Tablets are solid preparations each containing a single dose of one or more active ingredients and produced via compression of uniform volumes of particles. The secret behind the difference between each tablet is in the type of excipients used. Pharmaceutical industry use different excipients, diluents, disintegrants and lubricants to ensure that tablets of specified quality are prepared. However, one excipient can affect the properties of the tablets. Thus, it can be said that each excipient added has a specific role in the formulation of the oral tablets.

Pharmaceutical industry has always been facing obstacles in the manufacturing of drugs. The major obstacle that concerns the industry is the solubility of the active ingredient(s) [4]. This big challenge is important for the pharmaceutical scientists because active ingredients in tablets must undergo dissolution before they are available for absorption from the GI tract [5]. About 40\% of today's newly developed drugs are known to be hydrophobic and thus do not dissolve in water [6]. The first site for drug absorption is the stomach, where an oral administration can release its drug [4]. The rate of absorption is controlled by how fast the drug dissolves in the fluid at the site of absorption. Therefore, it can be said that the dissolution rate is the rate-limiting step in drug absorption [5]. 
During the past and current studies scientists have attempted several methods for enhancing dissolution rate of those poorly watersoluble drugs. Some of these methods are: a) formation of water-soluble complexes; b) reducing particle size to increase surface area; c) solubilization in surfactant systems [5]. Also, other attempts have been made such as: d) nanosuspensions; e) conversion of a crystalline molecule to its amorphous state; f) inclusion of cyclodextrins and liquisolid compacts. Among the mentioned techniques, liquisolid compacts is a promising method to increase aqueous solubility of hydrophobic drugs [7]. Although this paper and several other papers have shown successful results in the use of liquisolid compacts, there are not many liquisolid dosages available in market, as still liquisolid technique faces issues for poor powder flowability and compactability especially with high viscosity liquid vehicles. In this study, wet granulation that has not been previously used with liquisolid technique to improve powder flow, is now applied on liquisolid powder admixture to produce granules with improved flowability and tabletability. This makes large-scale production of those liquisolid compacts feasible and applicable to pharmaceutical industry. The concept of "liquisolid tablets" was evolved from the powdered solution technology where liquid medication can be used [1,8]. The term "liquisolid medication" implies to solid drugs dispersed in non-volatile solvent system with the addition of appropriate powder excipients such as carriers, coatings and disintegrants. Liquisolid compacts possess acceptable flowability and compressibility properties depending on the non-volatile liquid vehicle used that are mostly low viscosity liquid vehicles such as PEG. In this study high viscosity liquid vehicle with good enhancement dissolution properties for drugs will be applied with liquisolid technique with application of wet granulation before tablet compaction. The information on liquisolid technique was first introduced by Spireas [8]. Moreover, knowing the appropriate amounts of carrier and coating materials to produce maximum efficacy is very important. Therefore, several equations (Eq 1-4) are used to calculate the right amount of carrier, coating and liquid medication needed for the formulation of the liquisolid compacts, as explained in previous section.

$$
\mathrm{L}_{\mathrm{f}}=\Phi_{\mathrm{CA}}+\Phi_{\mathrm{CO}}(1 / \mathrm{R})
$$

Equation 1

Where $\Phi_{C A}$ is the flowable liquid-retention potential of the carrier

and $\Phi_{\mathrm{CO}}$ is the flowable liquid-retention potential of the coating material.

$$
\mathrm{L}_{\mathrm{f}}=\mathrm{W} / \mathrm{Q}
$$

Equation 2

Where $\mathrm{L}_{\mathrm{f}}$ is the "loading factor" where the maximum amount of drug liquid loads on the carrier material.

$\mathrm{Q}$ is the amount of carrier (MCC, PH102)

and $\mathrm{W}$ is the liquid medication (PEG/Synp/Cremophor)

$$
\mathrm{R}=\mathrm{Q} / \mathrm{q} \quad \text { Equation } 3
$$

The amount of Q can be calculated and applied to Equation 3 to calculate the amount of coating (Cab-o-Sil ${ }^{\circledR} \mathrm{M}-5$ (q)) required. Hence, after the calculation of $\mathrm{Q}$ and $\mathrm{q}$ the formulation of the liquisolid dosage can be initiated. In addition, R-value is the ratio between the carrier and the coating and it has been proposed that the ratio of 20 will produce a flowable and compactible admixture $[1,5]$.

Before using the above equation and before formulating the liquisolid compacts, determination of the optimal flowable liquidretention potential for the carrier and coating used is required, which is the $\Phi$ value required for equation [1]. This is usually the first step in the formulation. This measurement is termed as "Angle of slide", where it evaluates the flowability of carrier (MCC) and coating material (Silica). Then, a graph of angle of slide $(\theta)$ versus the measured $\Phi$ value is plotted and hence the load factor is then calculated from the results obtained. The $\Phi(\mathrm{Eq} 4)$ value is defined as "the maximum weight of liquid $\left(\mathrm{W}_{\text {liquid }}\right)$ that can be retained per unit weight of the sorbent $\left(\mathrm{W}_{\text {solid }}\right)$ yielding a mixture with acceptable flowability" [8]

$$
\Phi \text { value }=\mathrm{W}_{\text {liquid }} / \mathrm{W}_{\text {solid }}
$$

Equation 4

The liquisolid tablets that contain the hydrophobic drug are expected to show significant increase in wetting properties and surface area of the drug availability and thus, enhanced drug dissolution. Nevertheless, this new dosage form will benefit the pharmaceutical industry in offering the most efficient drugs that can then be released to market to improve patient compliance. This is because, the patient would need less dose and frequency and that is all due to the liquisolid tablets that release the hydrophobic drug without problems and at the same time making sure the drug reaches its maximum bioavailability.

The hydrophobic drug that had been used in this study is Glibenclamide. It is from a group of antidiabetic drug known as the sulfonylurea. This class of drug is known to be associated with some serious side effects such as hypoglycaemia. The drug comes in two doses: 2.5 and $5 \mathrm{mg}$. However, general practitioners mostly prescribe the $5 \mathrm{mg}$ tablets. In this study, liquisolid technique was used to formulate Glibenclamide into $4 \mathrm{mg}$ dose instead of $5 \mathrm{mg}$. Therefore, the aims of this study are to formulate free flowable and compressible liquisolid powder admixture of Glibenclamide using wet granulation technique jointly with liquisolid technique to enhance drug dissolution rate. 


\section{Material and Methods}

\section{Materials}

Glibenclamide was obtained from Sigma-Aldrich. The following excipients were provided by different companies: Microcrystalline cellulose (Avicel ${ }^{\circledR}$ PH102) (FMC Corp., Philadelphia, USA), colloidal silicon dioxide (Cab-o-sil ${ }^{\circledR}$ M-5) (cabot Corporation, Rheinfelden, Germany), potato starch (BDH laboratory supplies, Poole England), Polyethylene glycol 400 (Sigma-Aldrich, Poole, UK), (Synperonic PE/L44) (ICI surfactants, Everberg, Belgium), polyoxyl 35 castor oil (Cremophor ${ }^{\circledR}$ ELP) (BASF Aktiengesselschaft, Ludwigshafen, Germany), Polyvinylpyrrolidone (Sigma Aldrich) and magnesium stearate (BDH,UK). All materials used were of either analytical or pharmacological grade.

\section{Methodology}

Determination of the angle of slide for Avicel ${ }^{\oplus}$ PH 102 and Cab-o-sil ${ }^{\oplus}$ (fumed silica): Angle of slide is also referred to as the "optimal flowable liquid-retention potential" was measured to evaluate the flow properties of the excipients used: the angle of slide was obtained by the use of different liquid vehicles with Avicel PH102 and Silica. Initially, 10g of the carrier or the coating were weighed and then $2 \mathrm{~g}$ of the liquid vehicle (PEG400, Synperonic PE/L44 and Cremophor ${ }^{\circledast}$ ELP) were added slowly in increasing intervals. This admixture was then mixed and placed on a polished metal plate. The plate was tilted gradually until the admixture powder was about to slide. Then, the angle that was formed between the plate and the horizontal surface was known to be the "angle of slide $(\theta)$ " (Figure 1). This was repeated several times with different amount of liquids to observe the flow properties of the powder with the liquid vehicle. From there, the flowable liquid-retention potential ( $\Phi$ - value) was calculated of each liquid/powder admixture. With the aid of excel, the angle of slide (for optimal flow properties) corresponding to $33^{\circ}$ of a liquid/powder admixture represented the flowable liquid-retention potential of Avicel ${ }^{\circledR}$ and Silica ${ }^{\circledR}$.

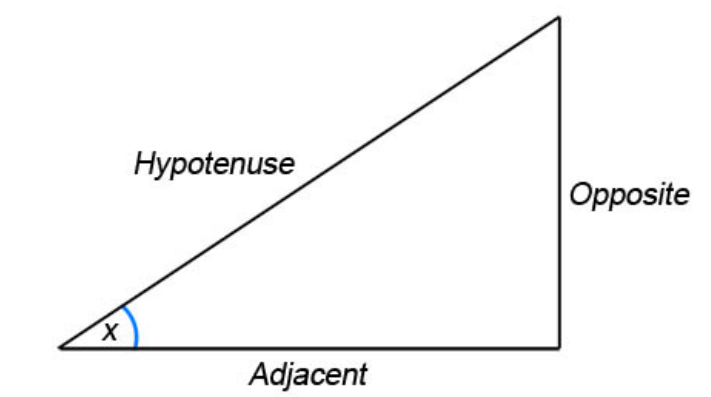

Figure 1: Diagrammatic representation of angle of slide. Note: $x=\theta$

$$
\operatorname{Tan}^{-1}(\theta)=\text { opposite/adjacent }
$$

Preparation of liquisolid powders: Glibenclamide liquisolid formulations denoted as G1 to G3 (Table 1) were prepared using PEG 400, Synperonic PE/L44 and Cremophor ELP as liquid vehicles, with 10\% drug concentration. From table 1, the exact amount of drug and excipients were measured using a class A balance. In the first step $4 \mathrm{mg}$ of Glibenclamide was dispersed in the liquid vehicle (PEG 400, Synperonic PE/L44 and Cremophor ELP) with continuous mixing using pestle and mortar. The mixing process was performed for around 5 min until no drug particles were seen undispersed. This was then followed by the gradual addition of the appropriate amount of carrier which was Avicel ${ }^{\circledR} \mathrm{PH} 102$. Again, the time of mixing was between 5 to 10 min until proper paste has been formed. Following that, the Silica, which acts as a coating material, was added to convert the wet mixture into dry powder under continuous mixing. The Avicel ${ }^{\circledR}$ and Silica ${ }^{\oplus}$ were used at a fixed powder ratio (R) 20 (refer to Eq 3). Finally, a 5\% w/w of potato starch which acts as a disintegrant was added into the admixture and mixed for 10 min. Then the magnesium stearate $0.75 \%$ was added at the end.

\begin{tabular}{|c|c|c|c|c|c|c|c|c|c|c|c|}
\hline $\begin{array}{l}\text { Liquisolid } \\
\text { system }\end{array}$ & $\begin{array}{l}\text { Non-volatile } \\
\text { liquid vehicle }\end{array}$ & $\begin{array}{c}\text { Drug } \\
\text { Concetration } \\
\text { in liquid } \\
\text { medication } \\
(\% w / w)\end{array}$ & $\begin{array}{l}\text { Carrier } \\
\text { Coating } \\
\text { (R) }\end{array}$ & $\begin{array}{l}\text { Liquid } \\
\text { load } \\
\text { factor } \\
\text { (Lf) }\end{array}$ & $\begin{array}{c}\text { Liquid } \\
\text { vehicle } \\
(\mathrm{mg})\end{array}$ & $\begin{array}{c}\text { Active } \\
\text { ingre- } \\
\text { dient } \\
(\mathrm{mg})\end{array}$ & $\begin{array}{l}\text { Carrier } \\
(\mathrm{Q})(\mathrm{mg})\end{array}$ & $\begin{array}{l}\text { Coating } \\
(\mathbf{q})(\mathrm{mg})\end{array}$ & $\begin{array}{c}\text { Disin- } \\
\text { tegrant } \\
\text { (maize } \\
\text { starch) } \\
(\mathbf{m g})\end{array}$ & $\begin{array}{l}\text { Lubrica } \\
\text { nt (mg) }\end{array}$ & $\begin{array}{l}\text { Unit } \\
\text { dose } \\
\text { (mg) }\end{array}$ \\
\hline G1 & PEG $400^{*}$ & 10 & 20 & 0.168 & 36.0 & 4.0 & 238.1 & 11.9 & 15.4 & 2.31 & 308.0 \\
\hline G2 & Synperonic PE/L44 & 10 & 20 & 0.266 & 36.0 & 4.0 & 150.4 & 7.5 & 10.5 & 1.6 & 210.0 \\
\hline G3 & Cremophor ELP & 10 & 20 & 0.52 & 36.0 & 4.0 & 76.9 & 3.8 & 6.4 & 0.96 & 128 \\
\hline Conventional & - & - & 20 & - & - & 4.0 & 238.1 & 11.9 & 13.5 & 2.0 & 270 \\
\hline
\end{tabular}

* PEG: polyethylene glycol

Table 1: Formulation of Liquisolid systems of Glibenclamide 
Granulation of Liquisolid powder admixture using PVP: The liquisolid powder was formed into granules using 10\% Polyvinylpyrrolidone (PVP). The PVP was prepared by weighing $10 \mathrm{~g}$ of PVP and $90 \mathrm{~g}$ of distilled water, and then it was placed in water bath at 60 degrees Celsius to dissolve the PVP in water. Furthermore, the required amount of PVP was added to the prepared liquisolid powder in intervals and mixed for approximately 2-3 minutes until a soft wet mass reached by feeling the wet mass. The correct moisture content was reached when the powder felt like sand from the beach in the morning. The next step was to perform the "snow ball experiment" [9], which is to take a hand full of wet mass and form a snowball like agglomerate and drop it into a tray from half a meter above the surface, when the agglomerate breaks into large pieces or only cracks, means the correct amount of moisture was added to the wet mass. Finally, the wet mass was then sieved using two mesh sizes $500 \mu \mathrm{m}-710 \mu \mathrm{m}$. The formed granules were placed to dry at a $45{ }^{\circ} \mathrm{C}$ for $120 \mathrm{~min}$. It is important to mention, that the granulation step was performed after the formulation of the liquisolid powder and before the compaction step, therefore, it was done as a final step before tablet formation.

Determination of flow and packing properties of the prepared liquisolid powders: Due to the poor flowability and compressibility properties of the ungranulated liquisolid powders, the packing properties of the prepared liquisolid powder were determined only for the granulated liquisolid compacts and for glibenclamide conventional formulation. The compaction force used was different between each liquisolid formulation due to difference in the ingredients and tablet weight. Therefore, the ungranulated liquisolid compacts were compacted manually due to its poor compactibility properties. The powder flow and packing properties of each prepared liquisolid powder was determined using tap volumeter (J. Engelsmann AG, Ludwigschafen, Germany). The liquisolid powder was weighed then poured into a $100 \mathrm{ml}$-measuring cylinder. The volume of the poured powder was recorded as $\mathrm{V}_{\mathrm{b}}$ and the tapped volume (after sufficient taps) which recorded to give a constant volume on a tap density apparatus was noted as $V_{t}$. Hence, from the recorded volumes, the density of the liquisolid formulation was calculated using the below (Eq 5) to give the bulk density $\left(p_{\mathrm{b}}\right)$ and tapped density $\left(p_{\mathrm{t}}\right)$ in $\mathrm{g} / \mathrm{mL}$.

$$
\begin{aligned}
& p_{\mathrm{b}}=\text { Weight of powder } / \mathrm{V}_{\mathrm{b}} \\
& p_{\mathrm{t}}=\text { Weight of powder } / \mathrm{V}_{\mathrm{t}}
\end{aligned}
$$

Equation 5

From there, the compressibility index (CI \%) of the formulations was calculated using equation [6] to investigate the flowability of the powders:

$$
\text { CI \% }=100 \mathrm{x}\left(\mathrm{p}_{\text {tapped }}-\mathrm{p}_{\text {bulk }}\right) / \mathrm{p}_{\text {tapped }}[10] \quad \text { Equation } 6
$$

According to the British Pharmacopoeia [11], the smaller the value of CI\% the better the flow properties of the powder. Thus, CI\% below $25 \%$ represents passable flow properties and values above $40 \%$ are indicative of poor powder flow.

Compaction of liquisolid granules and conventional tablets: The powders were then compacted manually (non-granulated liquisolid powder) or automatically (liquisolid granules) depending on its flow properties (mentioned in previous section) on a $10 \mathrm{~mm}$ flat-faced punch and die set using a single punch tabletting machine (type 3, Manesty Machines Ltd., Liverpool, UK). Different formulations had different compression force to produce acceptable hardness (4-6kg) depending on various factors in the formulation such as the tablet weight. For G3 (refer to Table 1). It is worth to mention that some formulations could not achieve this hardness (G2) with any compression force. Nevertheless, due to the small unit dose, extra avicel was added as filler to increase the tablet weight to $250 \mathrm{mg}$ unit dose and to get a stronger and bigger tablet. The extra Avicel was added in the final stage after the formation of liquisolid granules; therefore it did not affect the R-value of the formulation. Additionally, Glibenclamide conventional tablets were prepared by adding $4 \mathrm{mg}$ of the active ingredient and the rest of the excipients apart from the non-volatile liquid vehicle and hence compacted automatically.

\section{Characterisation of liquisolid formulations}

In-vitro dissolution studies: In vitro dissolution studies were performed for each batch of liquisolid formulation market drug (Wockhardt) and conventional tablets using USP dissolution apparatus II (Caleva Ltd., Dorset, UK). In this method distilled water was used as dissolution medium. The liquisolid tablets Glibenclamide placed in a dissolution medium of $900 \mathrm{~mL}$ at a temperature of $37 \pm 0.1{ }^{\circ} \mathrm{C}$ and stirred at a paddle speed of 100rpm. Moreover, ten millilitres samples, recommended by British Pharmacopoeia [11] were collected at intervals 5, 10, 15, 20, 25, 30, 45, 60 and $90 \mathrm{~min}$ and were replaced by $10 \mathrm{ml}$ equal amount of distilled water to maintain a constant volume. Furthermore, the collected samples of Glibenclamide were analysed spectrophotometrically at 229 $\mathrm{nm}$ for determination of Glibenclamide content using a calibration curve. The dissolution experiment was performed to compare the percentage drug release of liquisolid tablets compacted manually for non-granulated powders and compacted automatically for granulated powders, pure drug, market drug and conventional tablets.

Quality control tests of the prepared tablets: The liquisolid compacts and conventional tablets were evaluated via quality control tests in accordance to the British Pharmacopoeia specifications [11].

Uniformity of tablet weight (BP): Twenty tablets were randomly chosen from each formulation and weighed individually. The average mass of the twenty tablets was calculated. 
Tablet hardness: Six tablets were selected at random from each formulation of the two drugs and the force in Newton needed to crush them was examined using the hardness tester (Model 2E/205, Schleuniger and Co., Switzerland). In addition to that, before the test was performed, the tablets' diameter and thickness were measured.

Friability: The friability tester (FRV 1000, Copley Scientific, UK) was used to measure the friability of 10 randomly picked tablets from different formulation. The weight of the tablets was recorded before and after using the tester. The drum was rotating at $25 \mathrm{rpm}$ for $4 \mathrm{~min}$. The weight difference was recorded and the percentages of friability were calculated using equation [7].

$$
\% \text { Friability }=(\text { loss of mass/initial mass }) \times 100 \quad \text { Equation } 7
$$

Disintegration: The disintegration test was performed on six tablets from different formulations at $37 \pm 1^{\circ} \mathrm{C}$ in distilled water using the disintegration unit (Manesty Machines Ltd., Liverpool, UK). The time of disintegration of the tablet was recorded when there was no residue (soft mass with no palpably firm) in the cylindrical transparent tube, which indicated that the tablet has fully disintegrated.

\section{Statistical analysis}

One-way ANOVA and Post Hoc test were applied to see if there is any significant difference between formulations. Results are quoted as statistically significant when $P<0.05$.

\section{Results and Discussion}

\section{Determination of the angle of slide for Avicel PH 102 (MCC) and Cab-o-sil (fumed silica)}

It is vital to measure the angle of slide because it is the preferred method to determine the flowability of powders with particle size less than $150 \mu \mathrm{m}$ [8]. In this study, as stated by the manufacturer, the particle sizes of Cab-o-sil ${ }^{\circledR} \mathrm{M}-5 \mathrm{P}$ and Avicel ${ }^{\oplus} \mathrm{PH} 102 \mathrm{were}^{-}$ $133.8 \mu \mathrm{m}$ and $0.2-0.3 \mu \mathrm{m}$ respectively. In addition to that, Spireas et al [8] clarified that when the drug particle dissolved in the liquid vehicle, the liquid initially absorbed into the interior of the particles gets captured by its internal structure, thus adsorption of the liquid starts taking place onto the internal and external surfaces of the porous carrier particles after saturation state of this process has occurred [12]. Furthermore, the coating material, which has high adsorptive property and large surface area, gives the liquisolid system the desirable flow characterisation. Thus determination of the flow properties of the powder excipient and liquid/powder admixture is significant to ensure a success liquisolid formulation. In addition to that, the viscosity of the liquid vehicle plays an important role in the formulation of the liquisolid compacts. The viscosity of PEG 400 and Cremophor ELP are 105-130 and 650-800 $\mathrm{mPa}$ s, respectively. Tiong and Elkordy [1] reported that the viscosity of the liquid vehicle is inversely proportional to the amount of carrier and coating material required in a liquisolid formulation and accordingly the $\mathrm{L}_{\mathrm{f}}$ is increasing with increasing the viscosity of liquid vehicle. High viscosity liquid vehicle such as synperonic PE/L44 and cremophor ELP leads to poor powder flowability and compactibility of liquisolid admixture even with application of compressibility index as described by Spireas [8]. In addition, Suliman et al [13] studied the compressibility index of norfloxacin liquisolid powder using PEG and synperonic that required the application of an equation. However, this method is very time consuming especially that the equation needs to be understood thoroughly before application, which is not convenient for manufacturing purposes. Also, application of compressibility index requires several samples as a result loses excessive amount of powder before the liquisolid powders are compressed, which is again a great disadvantage to the pharmaceutical industry. Nevertheless, although the flowability of liquisolid powder using synperonic was enhanced using the compressibility index as done by Suliman et al [13], it is yet not a promising method for enhanced flowability of liquisolid admixtures using high viscosity liquid vehicles such as synperonic and cremophor. Accordingly, wet granulation was applied in this study to overcome these problems that will be beneficial for large-scale production in pharmaceutical industry.

\section{Determination of flow and packing properties of the prepared liquisolid powders-Discussion}

The determination of the powder flowability is important in handling, packing, mixing and compression. In addition to that, the major obstacle pharmaceutical industry face is the poor flow properties of powders. This major problem affects the uniformity of flow out of the hopper and further problems in compaction [1]. Because, uniform and reliable flow from the hoppers into the die of the compressing machine, ensures uniform tablet weight and drug content. There are several factors that can affect the flow properties of the liquisolid powder, which are physical, environmental as well as mechanical factors. Therefore, the Carr's compressibility index was calculated for each liquisolid powder to determine its flow properties. This means the flow properties are affected by particle shape, particle size, porosity, density, moisture content and surface roughness. Additionally, Carr's compressibility index is a good parameter in reflecting interparticle friction. The calculated Carr's compressibility index of Glibenclamide liquisolid granules is presented in Table 2. It is a good parameter to evaluate the flow of the prepared powder. The lower the Carr's compressibility index means the better the flow of the powder. In this study, as shown in Table 2, the results for G1 and G3 and conventional liquisolid powders were $12.6 \%, 8 \%$, and $18 \%$, respectively, all have CI below $25 \%$. On the other hand, G2 
result showed poor flowability with CI\% being above 25\% (28\%). This can be related to the amount, type and property of the liquid vehicle used (PEG400, Synperonic Pe/L44 and cremophorELP). Nevertheless, the flowability results obtained were only for granulated liquisolid powder. This is due to the poor flowability properties (which were obvious visually) of the liquisolid powder before granulation using PEG400, Synperonic PE/L44 and cremophor ELP (viscous liquid vehicle). Thus, this shows that the properties of the liquid vehicle can interfere with the flow property of the formulation making it better or poor flowing.

\begin{tabular}{|c|c|c|}
\hline $\begin{array}{c}\text { Carr's index }(\mathbf{C l} \%) \\
\text { of prepared liquisolid powder granules of glibenclamide }\end{array}$ & \\
\hline Liquisolid system & Carr's index $(\mathrm{Cl} \%)$ & Type of Flow \\
\hline G1 & 12.6 & Excellent \\
\hline G2 & 28.0 & Poor \\
\hline G3 & 8 & Excellent \\
\hline Conventional & 18 & Fair \\
\hline
\end{tabular}

${ }^{*}$ For the composition of each formula refer to Table 1 .

Table 2: Carr's compressibility index of Glibenclamide liquisolid granules and conventional powder

Moreover, in addition to the coating properties of Cab-o-sil, it can also act as a glidant (concentration of 0.1-0.5 \%) to improve powder flowability. Furthermore, these liquisolid powders were compacted electronically into tablets. It can be seen from Table 2 , that the liquisolid granule formulation using cremophor ELP showed best flowability outcome due to the addition of excess avicel (carrier) that was added to increase the weight of the tablet to $250 \mathrm{mg}$ unit dose. Therefore, it is clearly visible that granulation with the aid of excess avicel has enhanced the flowability properties of the liquisolid powder using one of the high viscous liquid vehicle i.e. cremophor ELP. On the other hand, liquisolid powders using all three vehicles used in this study were not compacted automatically unless formed into granules. This is due to poor flowability and compressibility properties of the liquisolid powder and due to the elasticity properties of the liquid vehicle used. Elkordy et al [14] prepared two liquisolid formulations using Synperonic PE/L61 with Solutol HS 15 (1:1) at both concentrations 10\% and 30\% which were incompactible, and hence no tablets were formed. The powder deformed upon compression and returned to its original shape after removal of the compaction force. This is clearly seen in the case of glibenclamide liquisolid with cremophor ELP (Figure 2a). Compression of ungranulated powder admixture was impossible due to the elastic behavior of the liquisolid formulation. As it is seen in Figure 2a, the powder not only was not compressed into tablet, but also formed a compressed unwanted shape, which is not desirable in the industry. Therefore, granulation was used to overcome this problem. After using granulation, the powder was compressed automatically into tablets with no capping or cracking which complied with quality control test of the British Pharmacopeia [11]. In a concentrated suspension, weak Van der Waal forces hold flocculated particles together. This often results in plastic flow [14,15]. If the shear stress is low, such materials might possess elastic behavior. However, when the maximum yield stress is exceeded where the Van der Waal forces are broken, plastic flow is exhibited. There must be a balance for plasticity. Although plastic deformation helps the solid links to form between particles within the powder mix, excessive plasticity leads to the formation of tablets with high extent of hardness. The powder mix produced with Avicel and the liquid vehicle takes advantage of the elastic properties of the liquid vehicle and the plastic properties of Avicel, leading to the formation of a powder mix that is mainly elastic [14]. Thus, only few ungranulated liquisolid tablets were manually compressed for the purpose of dissolution comparison test study. As shown in Table 2, liquisolid granules using cremophor ELP (G3) showed best flowability outcome compared to PEG 400 (G1). According to Tiong and Elkordy [1], liquisolid naproxen showed best flowability with PEG 400 and not with cremophor EL (poor flowability). This is due to the high viscosity behaviour of the non-volatile liquid and due to load factor being above 0.25 [7]. The higher the liquid load factor the poorer the flowability characteristics of the liquisolid powder (see Table 1 for details). This is because there will be fewer amounts of carrier and coating available in the formulation and excess amount of liquid vehicle being available leading to agglomerates [14]. Also, in this current study, synperonic (G2) showed poor flowability compared to the conventional powder (fair flow) but both were automatically compressible. This is clearly linked to the amount of liquid vehicle present that delays the flow of the powder. According to Javadzadeh et al. [5], using HPMC or PVP as microsystems can improve flowability.

It is known that for liquisolid tablets [8], the determination of compressible load factor is important parameter. However, this parameter was not considered in this study and the liquid load factor was the only considered parameter) because its time and equation require long process. On the other hand, the new technique of wet granulation was used instead. It has shown enhanced flowabiity of liquisolid powders that contain very viscous liquid vehicles such as cremophor ELP. Not only it enhanced the flowability but also allowed for automatic compression of the liquisolid granules, which was impossible, to achieve without granulation. Also, the excipients used played a great role in the flowability and compactibility of the liquisolid powder. For example, microcrystalline cellulose (MCC, Avicel ${ }^{\circledR}$ PH102), is a widely used excipient in pharmaceutical tableting, because of its good compactible properties. 


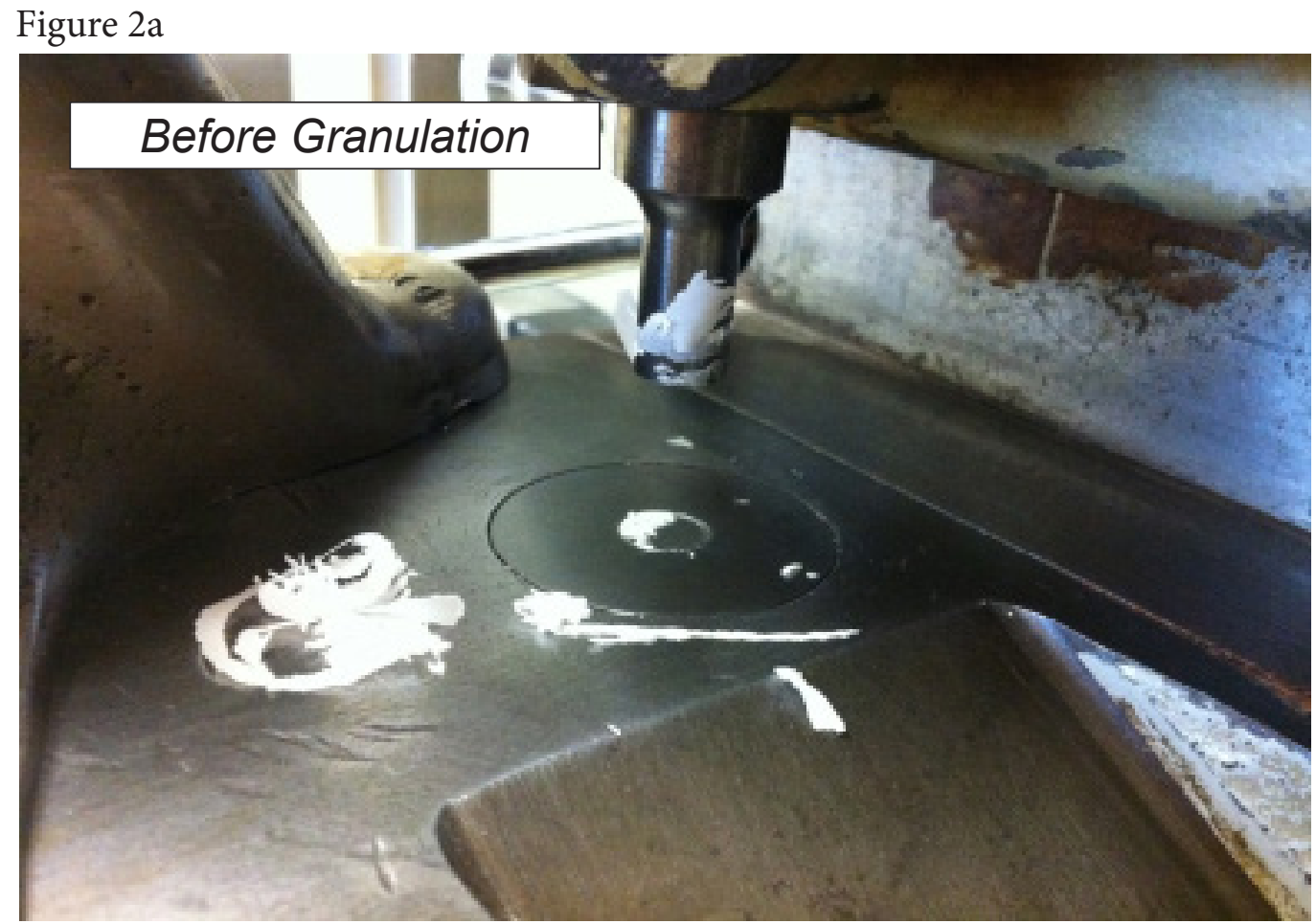

\section{Figure $2 b$}

\section{Before Granulation}

Figure 2: (a) Glibenclamide and 10\% CremophorELP powder without granulation, (b) Glibenclamide and10\% CremophorELP powder without granulation 
This can be explained by the chemical properties of the MCC. The microcrystalline particles are held together with hydrogen bonds, which in return affect the strength and cohesiveness of the tablet $[1,14]$. Therefore, MCC was used as a filler to increase the tablet size to a medium size tablet of 250mg unit dose. By the addition of MCC the tablets were compressed evenly without any cracking or capping. Nevertheless, Glibenclamide and cremophor ELP 10\% without granulation were difficult to compress neither manually nor automatically but they became elastic and showed an unexpected outcome, they were poorly flowable and were cohesive as seen in Figures $2 \mathrm{a}$ and $2 \mathrm{~b}$. Therefore pre-granulation liquisolid powders did not undergo flowability test. On the other hand, as seen in Figure $3 \mathrm{a}$ and $3 \mathrm{~b}$, the granulated liquisolid powder of Glibenclamide using cremophor ELP showed better shape with enhanced flowability and thus compactible tablets.

So granulation is a successful technique to convert incompactible elastic powder into good flowability and compactibility powders with excellent flowability according to Carr's compressibility index.

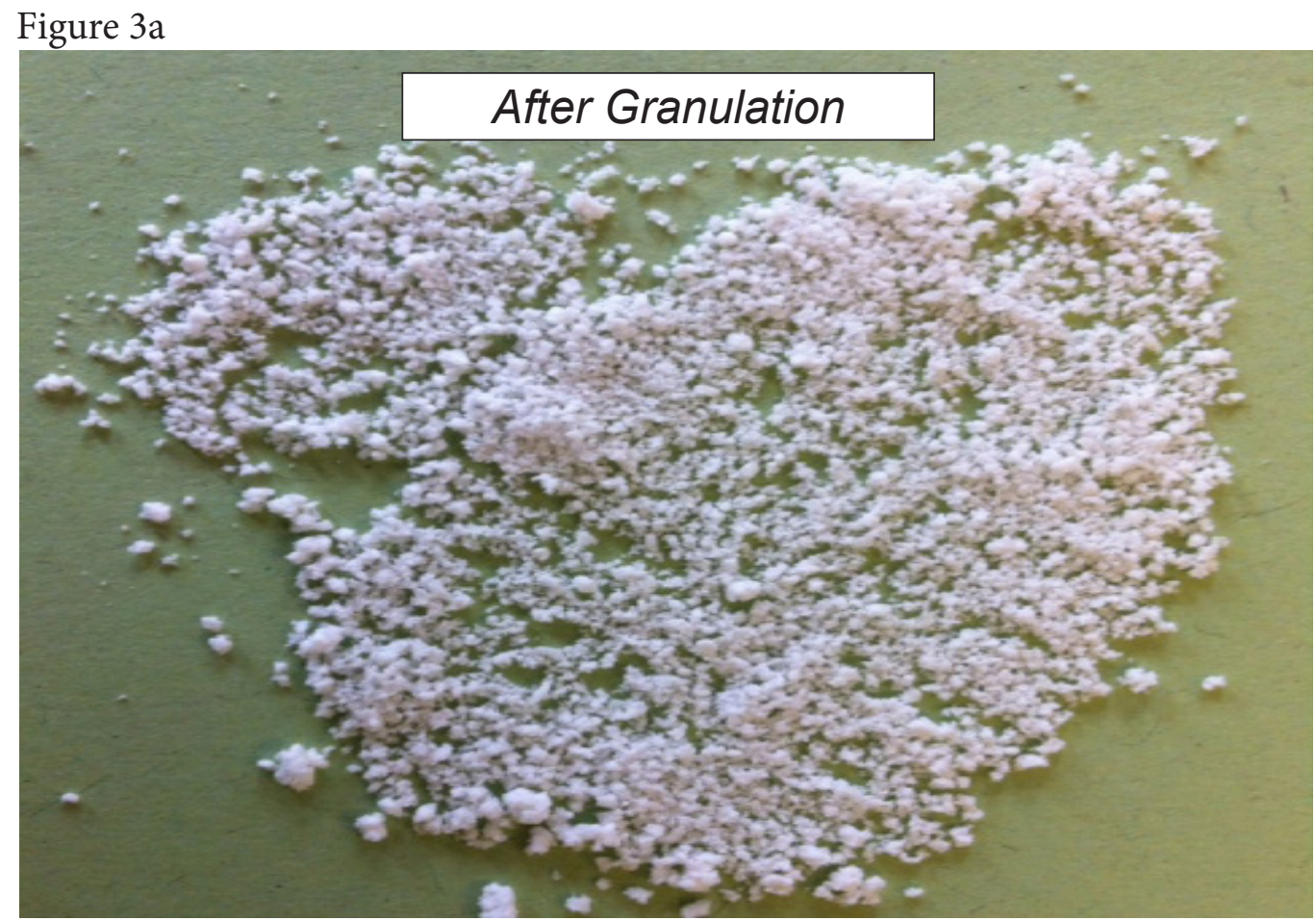

Figure $3 b$

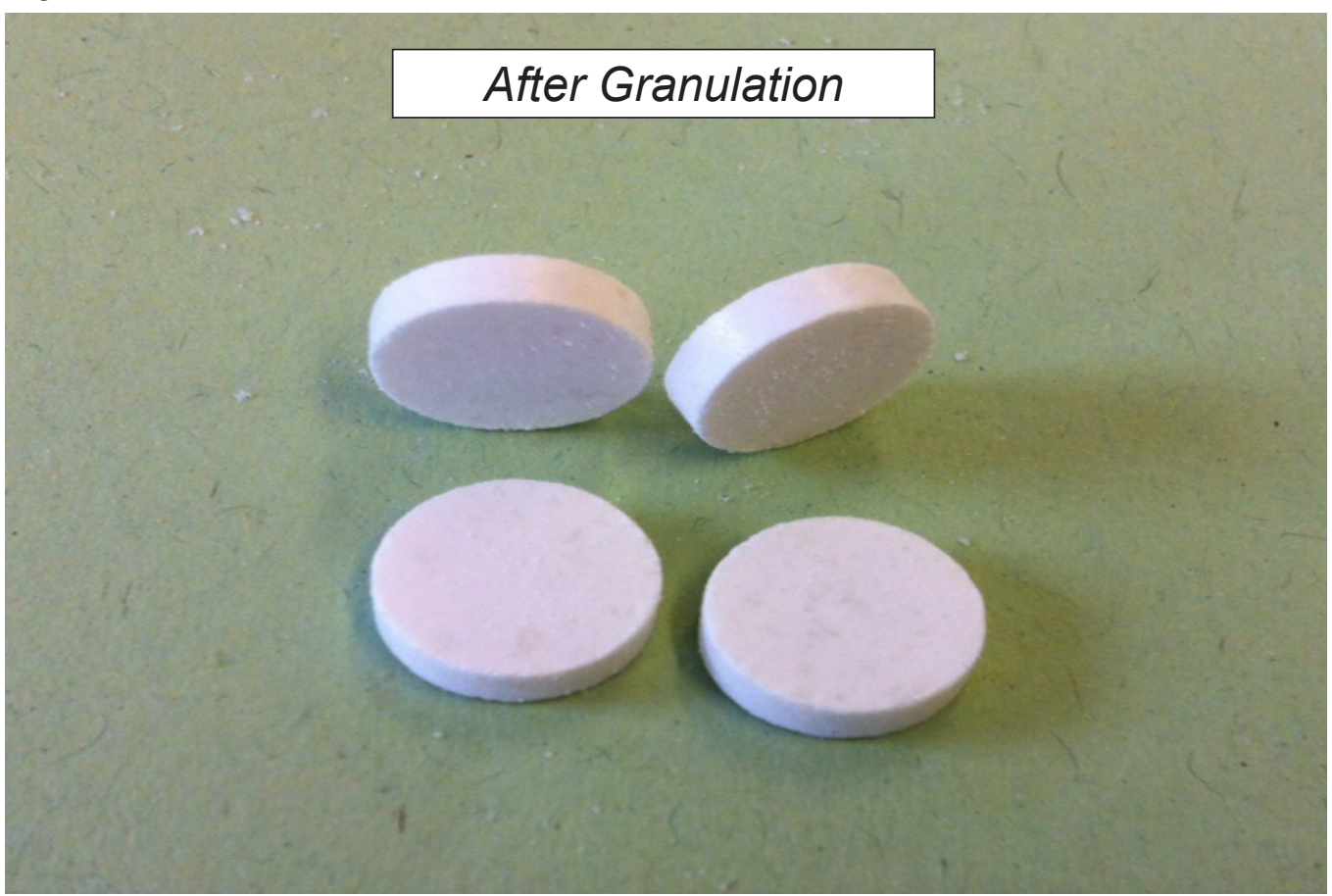

Figure 3: (a) Glibenclamide and 10\% CremophorELP granules after granulation, (b) Glibenclamide and 10\% CremophorELP tablets after granulation 


\section{Characterisation of liquisolid formulations}

In-vitro dissolution studies: The dissolution profiles of glibenclamide formulation were performed in distilled water only to study the effect of liquisolid technique on aqueous dissolution enhancement of the drug. The dissolution profile of Glibenclamide with $10 \%$ cremophor (Figure 4) shows highest drug dissolution rate $(\mathrm{P}<0.05)$ when compared to most formulations. Dissolution data for the pure drug showed no (or zero) drug release therefore its profile is not shown in Figure 4. Also, the liquisolid glibenclamide with granulation showed higher drug release than without granulation $(\mathrm{P}<0.05)$. It is seen in Figure 4, where G1, G2 and G3 (refer to Table 1 for composition) showed a higher drug release when granulation step was performed and inversely, the drug release was to a minimum when manual compaction was performed in the non-granulated form. The proposed suggestion is that liquisolid powders prior to granulation were compacted manually; therefore the force applied was relatively high making the pore size between particles comparatively small; hence the dissolution rate was reduced. However, when wet granulation was applied, automatic compaction was performed on liquisolid powder, resulted in lower force being applied and hence larger pores were formed and this allowed more dissolution medium, water, to penetrate through particles due to larger pore size.

Consequently, it can be concluded that the in vitro dissolution of liquisolid tablets is greatly influenced by granulation and by other three factors, which are: the type of vehicle, the drug-vehicle ratio and type of disintegrant [2,5]. In addition to that, some carriers could have an effect on the drug dissolution, for example in this case, microcrystalline cellulose was used and from previous research it showed that MCC has disintegration property that could facilitate disintegration of tablets and thus dissolution of drug [5]. In addition, a study by El Gizawy [16], suggested that single disintegrant has no significant effects on drug dissolution. Also, for explaining the dissolution characterisation and the percentage drug release of the formulation better, the Noyes-Whitney equation was used.

$$
\mathrm{dC} / \mathrm{dt}=\frac{\mathrm{k} \cdot \mathrm{A}(\mathrm{Cs}-\mathrm{Cb})}{\mathrm{h}}
$$

where $\mathrm{dC} / \mathrm{dt}$ is the dissolution rate of the drug particle; " $\mathrm{K}$ " is the diffusion coefficient of the dissolved drug particles, which are affected by the viscosity of the dissolution medium; "A" the surface area exposed to dissolution; "Cs" the saturation solubility of the drug in the diffusion layer; "Cb" is the concentration of drug in the bulk solution and " $\mathrm{h}$ " is the thickness of the diffusion layer around each drug particle [17]. Moreover, all dissolution tests were done under same paddle speed of 100rpm and same dissolution medium, which was, distilled water. Therefore it is assumed that the $\mathrm{h}$ and $\mathrm{K}$ are constant. Thus the factors that will affect the dissolution outcomes of the liquisolid compacts are A and (Cs - $\mathrm{Cb}$ ). Additionally, there are some factors that can affect the solubility of the solid drug in the dissolution medium such as temperature (endothermic or exothermic), dissolution medium (acidic or neutral), crystalline structure, presence of solubilising agents and molecular structure of the solute. In this study, PEG behaved with Glibenclamide differently when compared to other drug in other literature as it showed enhanced dissolution [5], but with Glibenclamide no great effect (Figure 4). Therefore, not all liquisolid vehicles work to the same extent with all hydrophobic drugs. Therefore, what could have affected the outcome is the presence of other compounds, which are the non-volatile liquid vehicles. As a result, each formulation had different dissolution profile depending on its liquid vehicle and its wetting property $[18,19]$. Basically, enhanced dissolution rate seen in liquisolid formulations is due to the mechanical treatment with the hydrophilic liquid vehicle, which increased the contact between the drug, and the dissolution medium which was distilled water [20-22]. In other words, surfactants increase the wettability of the drug making them more available for water molecules to penetrate and thus increase the drug dissolution and improve the absorption from the GI tract.

To relate the results to the equation, when formulating the liquisolid tablet, the drug particle was dispersed in the hydrophilic liquid vehicle which enables the wettability of the drug particles to increase, thus allowing the surface area of the drug particles which are available for dissolution increase too. The liquisolid system would increase the saturation solubility (Cs) of glibenclamide, due to the presence of that hydrophilic liquid vehicle yielding a higher dissolution profile. Moreover, the reason behind one formulation having higher dissolution rate than another is due to the solubility effect of the liquid vehicle itself. In other words, if the drug was soluble in that liquid then it can be predicted, that formulation will have higher dissolution profile. On the other hand, comparing drug release from market tablets and conventional tablets, the extent of the drug release for both of them was about $15 \%$ (Figure 5) and only $10 \%$ of the drug were released after 10 minutes, although, bearing in mind, market tablets contain 5 mg drug and conventional tablets contain $4 \mathrm{mg}$ of Glibenclamide. Also, for PEG 400 containing liquisolid formulations, the drug release was lower (however not significant $\mathrm{P}>0.05$ ) in the case of ungranulated compacts prepared manually. Presence of Synperonic and Cremophor enhanced Glibenclamide release better than PEG 400 did. Hence, PEG 400 is not a good choice of liquid vehicle to enhance Glibenclamide dissolution.

Interestingly, Cremophor ELP containing liquisolid compacts which prepared after granulation resulted in the highest drug release ( $>50 \%$ after $20 \mathrm{~min}$ ) (Figure 5), the same formulation without the wet granulation step could not be compacted into tablets even manually (Figure 2a). On the other hand, addition of Avicel to Cremophor ELP containing formulation to increase tablet volume (up to $250 \mathrm{mg}$ ), has no significant effect $(\mathrm{P}>0.05)$ on the drug release (Figure 4 ). Therefore, it can be said that wet granulation step has shown an advantage to the liquisolid formulation in the release of the hydrophobic drug and hence enhancement of the dissolution rate. 


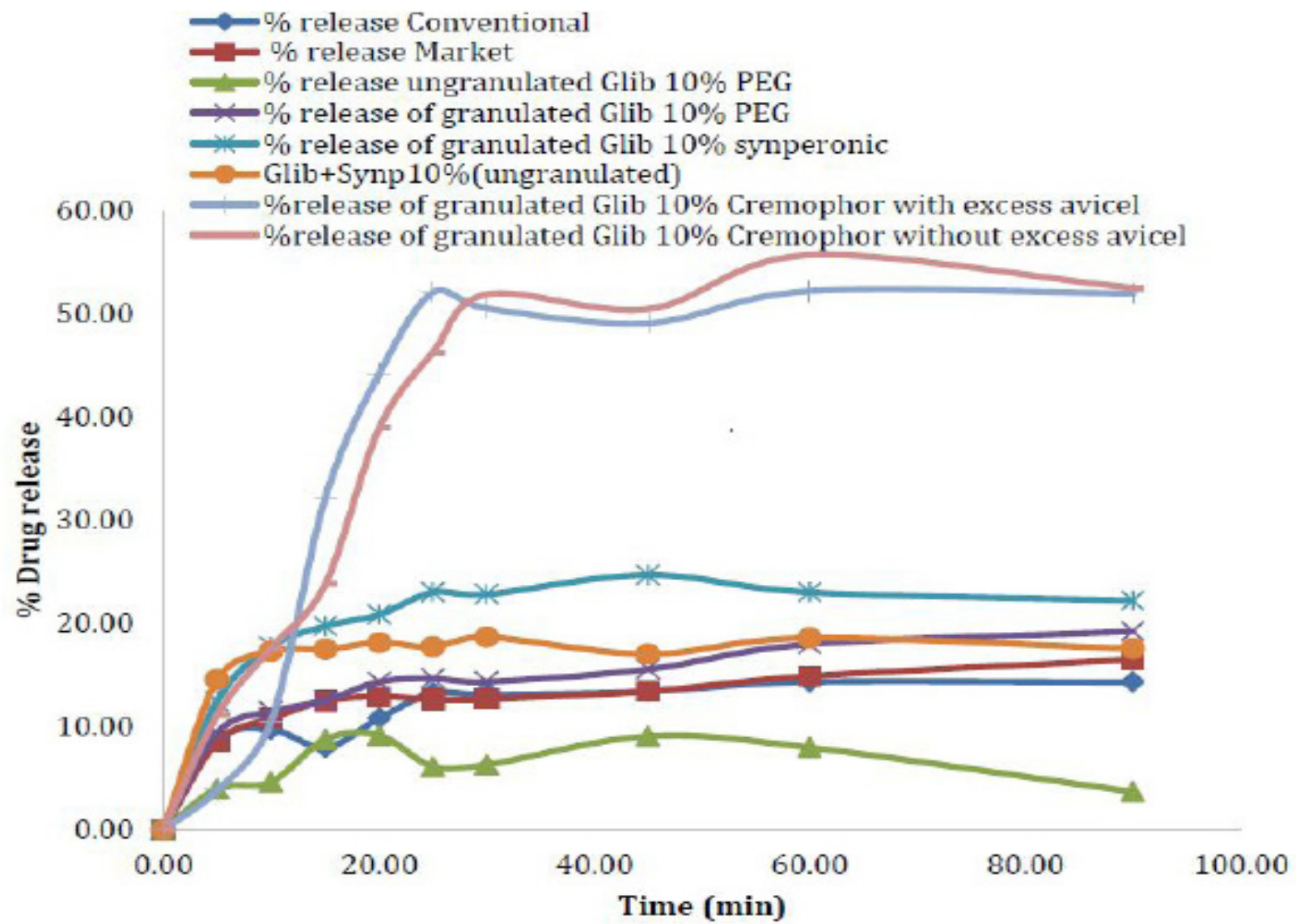

Figure 4: Dissolution profile of liquisolid compacts (G1-G3), market drug and conventional tablets in distilled water media. Error bars are standard deviations. See Table 1 for composition

Uniformity of tablet weight (BP): The permitted weight variation in BP, not more than two tablets differ from the mean by more than $5 \%$ if the average weight of the tablet was $250 \mathrm{mg}$ or more, and for less than $250 \mathrm{mg}$ the percentage deviation is $7.5 \%$. In this case, all prepared formulations including the conventional tablet did comply with British Pharmacopeia [11] specifications. The above uniformity of weight test was performed only on liquisolid tablets with granulation whilst non-granulated tablets were not tested for any quality control measures due to the manual compression and some complete incompression of the liquisolid powders.

Tablet hardness: Generally, ideal tablet hardness should be produced without applying excessive compression force where rapid tablet disintegration and drug dissolution are maintained at the same time. There are several factors tablet hardness depends on such as particle size, interparticle force and most importantly compression force. Usually, as compression force increased, tablet hardness and fracture resistance increased as well. In addition to that, El-Say et al. [18] found that there is a relationship between liquid load factor and the hardness of the tablets in the formula having approximately the same powder excipient ratio. Additionally, when liquid load factor increased, the hardness of the tablet decreased. However, this was not seen in this study, the data obtained showed no relationship to the liquid load factor of the liquid vehicle used. Our suggestion is that not only the liquid load factor has an effect on the hardness of the tablet, but also granulation process that was performed before compaction (refer to Table 3 for details). For G2 formulation, although hardness was small (1.8kg), but the tablet complied with friability test (<1\%).

Friability and Disintegration: All liquisolid tablets complied with BP friability test limits $(<1 \%)$, see Table 3 . The presence of a non-volatile liquid vehicle is expected to delay the disintegration time of the tablet. However, the presence of MCC speeds the process of disintegration giving a low disintegration time [5]. Disintegration time can be affected by the type of liquid vehicle used and other properties, such as tablet porosity and pore structures. Studies from literature suggest that there is a relationship between tablet hardness and disintegration time. Basically, when the hardness increases, the disintegration time increases [23]. In other words the higher the crushing force the longer the disintegration time will be. However, the above finding was not achieved in this study as the tablet hardness does not play a role in disintegration time (Table 3 and Figure 5). Nevertheless, all Glibenclamide liquisolid tablets including the conventional tablet did comply with BP specification of disintegration time. 


\begin{tabular}{|c|c|c|}
\hline Liquisolid system & Friability weight & Crushing Load (kg) \\
\hline G1 & $0.60 \%$ & 5.93 \\
\hline G2 & $0.40 \%$ & 1.82 \\
\hline G3 & $0.06 \%$ & 3.88 \\
\hline Conventional & $0.6 \%$ & 5.3 \\
\hline
\end{tabular}

* For the composition of each formula refer to Table 1 .

Table 3: Friability and hardness results of Glibeneclamide liquisolid granulated tablets including conventional tablet

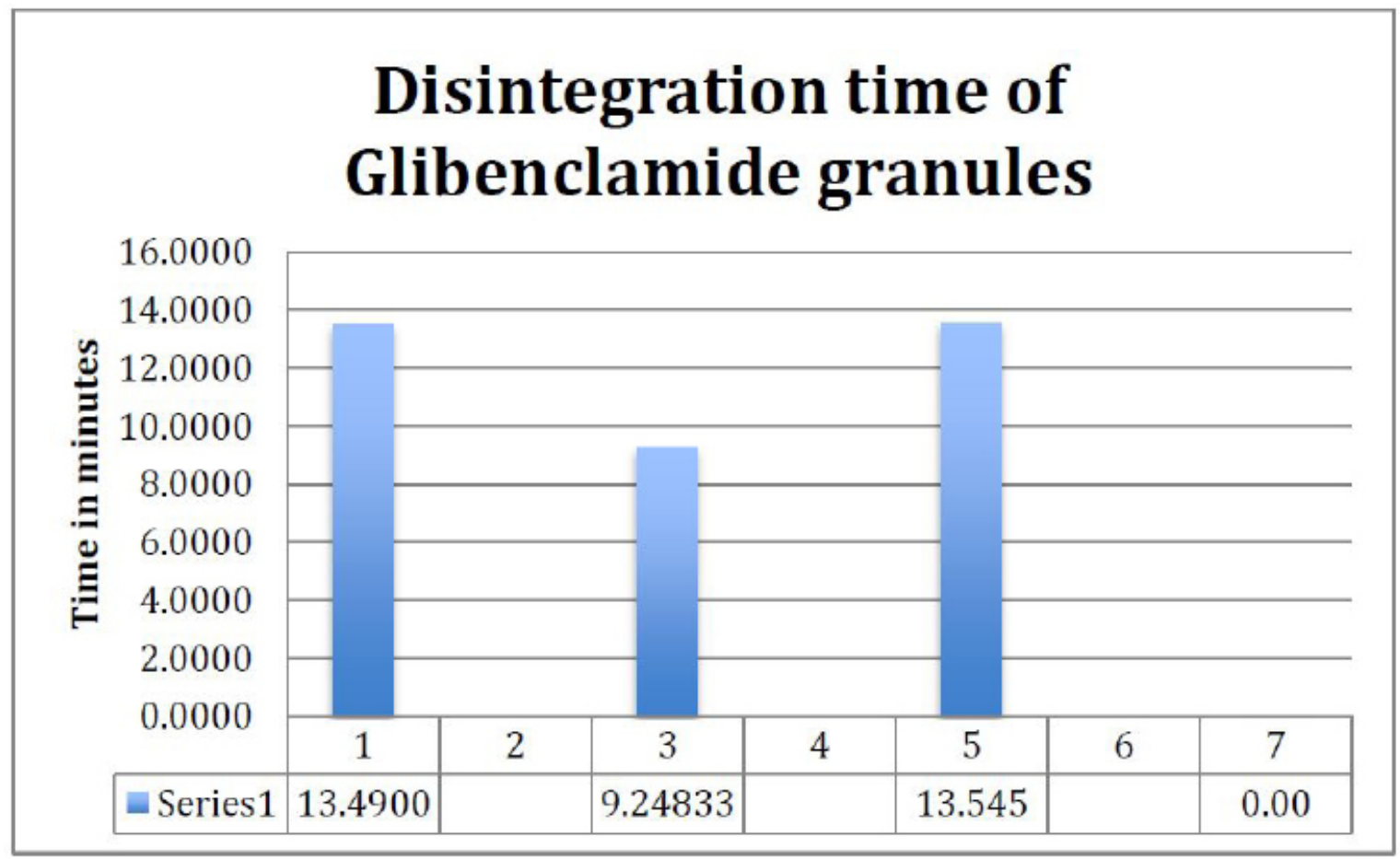

Figure 5: Average disintegration profile of Glibenclamide liquisolid granules with different excipients. (Refer to table 1 for composition). Note: $1=$ $\mathrm{G} 1,3=\mathrm{G} 2,5=\mathrm{G} 3$ and $7=$ Conventional tablets

\section{Conclusion}

Liquisolid technique changes the properties of Glibenclamide by inclusion of the drug particles (10\%w/w) in non-volatile liquid vehicles (PEG 400, Synperonic ${ }^{\circledR}$ PE/L44 and Cremophor ELP). Wet granulation of prepared liquisolid powders successfully produced automatically compressed tablets using the above-mentioned liquid vehicles. The best dissolution enhancement was seen with combination of Cremophor ELP with Glibenclamide $(10 \% \mathrm{w} / \mathrm{w})$. The viscous behavior of Cremophor ELP makes the flowability and automatic compression of liquisolid powders containing Cremophor very challenging. However, using the new technique performed in this study (wet granulation), those problems were resolved and well compressed tablets, which all complied with B.P. quality control tests, were produced. The enhanced drug dissolution by using liquisolid technique is a result of increasing surface area of drug particles and improving drug wetting properties and hence the large-scale industrial production for those formulations is more feasible. Therefore, it can be concluded, that the problem with liquisolid compacts, which is flowability and compressibility, has been resolved by the introduction of wet granulation system to liquisolid powder admixture before compression. Not only the dissolution of the hydrophobic drug is enhanced, but also the wet tablets are formed into strong well-compacted tablets with no lamination or capping. Accordingly by this, liquisolid system can use any type of viscous liquid vehicles in its composition and by application of wet granulation technique liquisolid powders can be compacted into tablets.

\section{References}

1. Tiong N, Elkordy AA (2009) Effects of liquisolid formulations on dissolution of naproxen. Eur J Pharm Biopharm 73: $373-84$.

2. Nokhodchi A, Javadzadeh Y, Siahi-Shadbad MR, Barzegar-Jalali M (2005) The effect of type and concentration of vehicles on the dissolution rate of a poorly soluble drug (indomethacin) from liquisolid compacts. J Pharm Pharm Sci 8: 18-25.

3. York P (2013) Design of dosage forms Aulton's Pharmaceutics: The design and manufacture of medicines ( $4^{\text {th }}$ edn) Churchill Livingstone, London.

4. Dressman J, Reppas C (2007) Drug solubility: how to measure it, how to improve it. Advanced Drug Delivery Reviews 59: 531-2. 
5. Javadzadeh Y, Siahi MR, Asnaashari S, Nockodchi A (2007) Liquidsolid technique as a tool for enhancement of poorly water-soluble drugs and evaluation of their physiochemical properties. Acta Pharm 57: 99-109.

6. Lipinski C (2003) Aqueous solubility in discovery, chemistry, and assay changes Drug Bioavailability: Estimation of Solubility, Permeability, Absorption and Bioavailability, Wiley-VCH Publisher, Gemany 18: 215-231.

7. Javadzadeh Y, Siahi-Shadbad M, Barzegar-Jalali MR, Nokhodchi A (2005) Enhancement of Dissolution Rate of Piroxicam Using Liquisolid Compacts. Farmaco 60: 361-5.

8. Spireas SS, Jarowski CI, Rohera BD (1992) Powdered Solution Technology: Principles and Mechanism. Pharm Res 9: 1351-8.

9. Leuenberger H (2011) Manufacturing Pharmaceutical Granules: Is the Granulation End Point a Myth? Granulation Workshop 1: 1-2.

10. Carr RL (1965) Evaluation flow properties of solids. Chem Eng 72: 163-168.

11. British Pharmacopoeia (2011) Electronic version.

12. Fahmy RH, Kassem MA (2008) Enhancement of famotidine dissolution rate through liquisolid tablets formulation: In vitro and in vivo evaluation. Eur J Pharm Biopharm 69: 993-1003.

13. Suliman AS, Anderson RJ, Elkordy AA (2014) Norfloxacin as a model hydrophobic drug with unique release from liquisolid formulations prepared with PEG200 and Synperonic PE/L-61 non-volatile liquid vehicles. Powder Technology 257: 156-67.

14. Elkordy AA, Tan XN, Essa EA (2013) Spironolactone release from liquisolid formulations prepared with Capryol ${ }^{\text {Ts }} 90$, Solutol ${ }^{\circledR} \mathrm{HS}^{-15}$ and $\mathrm{Kollicoat}^{\circledR}$ SR $30 \mathrm{D}$ as non-volatile liquid vehicles. Eur J Pharm Biopharm 83: 203-23.

15. Mosharraf M, Nystrom C (1995) Solubility Characterisation of practically insoluble drugs using the Coulter counter principle. Int J Pharm $122: 57-67$.

16. El-Gizawy S (2007) Effect of formulation additives in the dissolution of Meloxicam from Liquisolid tablets. Eqyp J Biomed Sci 25: 3-14.

17. Aulton ME (2013) Dissolution and solubility Aulton's Pharmaceutics: The design and manufacture of medicines, (4 ${ }^{\text {th }}$ edn) Churchill Livingstone, London.

18. EL-Say KM, Samy AM, Fetouh MI (2010) Formulation and Evaluation of Rofecoxib liquisolid tablets. Int J Pharm Sci Revi Res 3: $135-42$.

19. Akinlade B, Elkordy AA, Essa EA, Elhagar S (2010) Liquisolid Systems to Improve the Dissolution of Furosemide. Sci Pharm 78: 325-44.

20. Yadav VB, Yadav AV (2010) Enhancement of Solubility and Dissolution rate of BCS class II pharmaceuticals by nonaquious granulation technique. Int J Pharm Res Dev 1: 1-12.

21. Gubbi S, Jarag R (2009) Liquisolid Technique for Enhancement of Dissolution Properties of Bromhexine Hydrochloride. Research J Pharm Tech 2: 382-6.

22. Tayel S, Soliman II, Louis D (2008) Improvement of dissolution properties of Carbamazepine through application of the liquisolid tablet technique. Eur J Pharm Biopharm 69: 342-7.

23. Kitazawa S, Johono I, Ito Y, Teramura S, Okada J (1975) Effect of hardness on the disintegration time and the dissolution rate of uncoated caffeine tablets. J Pharm Pharmacol 27: 765-70. 